Tohoku J. Exp. Med., 2010, 222, 237-242

\title{
High Prevalence of Bronchiectasis in Adults: Analysis of CT Findings in a Health Screening Program
}

\author{
Hyun Jung Kwak, ${ }^{1}$ Ji-Yong Moon, ${ }^{1}$ Yo Won Choi, ${ }^{2}$ Tae Hyung Kim, ${ }^{1}$ \\ Jang Won Sohn, ${ }^{1}$ Ho Joo Yoon, ${ }^{1}$ Dong Ho Shin, ${ }^{1}$ Sung Soo Park ${ }^{1}$ and \\ Sang-Heon Kim ${ }^{1}$
}

${ }^{1}$ Department of Internal Medicine, Hanyang University College of Medicine, Seoul, Korea

${ }^{2}$ Department of Radiology, Hanyang University College of Medicine, Seoul, Korea

\begin{abstract}
Bronchiectasis is one of the common chronic respiratory diseases and associated with respiratory morbidity and mortality. However, neither its prevalence nor its etiology is well-defined. We aimed to estimate the prevalence and risk factors of bronchiectasis in adults. In a retrospective study, we analyzed radiologic findings on chest computed tomography (CT) images performed as part of a health-screening program. From January to December 2008, 1,409 (24.6\%) of 5,727 participants in the screening program of a health promotion center at a university hospital underwent chest CT scans based on the subject's decision. Bronchiectasis was diagnosed, if there was abnormal bronchial dilatation in any area of both lungs on chest CT. Respiratory symptoms, smoking status, and past medical history were also analyzed to define clinical characteristics and risk factors of bronchiectasis. Of 1,409 patients (aged 23-86 years), who were screened for respiratory diseases using chest CT for one year in a health promotion center, 129 patients $(9.1 \%)$ were diagnosed with bronchiectasis. The prevalence of bronchiectasis was higher in females than in males $(11.5 \%$ vs. $7.9 \%, p=0.022)$ and increased with age. Respiratory symptoms were reported in $53.7 \%$ of subjects. Previous history of tuberculosis (TB) (OR 4.61, 95\% Cl 2.39-8.88, $p=0.001)$ and age (OR 2.49, 95\% Cl 1.56-3.98, $p=0.001$ ) were significantly associated with bronchiectasis. This retrospective analysis of chest $\mathrm{CT}$ findings in health screening examinees revealed a very high prevalence of bronchiectasis in adults. Previous TB infection is one of the major causes of bronchiectasis.
\end{abstract}

Keywords: bronchiectasis; prevalence; chest CT; tuberculosis; risk factors

Tohoku J. Exp. Med., 2010, 222 (4), 237-242. ㅇ 2010 Tohoku University Medical Press

Bronchiectasis is a chronic airway disease characterized by permanent abnormal dilatation of bronchi and destruction of bronchial walls (Barker 2002). While there are many idiopathic cases, the common etiologies of bronchiectasis include postinfectious causes, genetic diseases, immune deficiency, and rheumatoid arthritis. Many patients present with chronic cough that produces mucopurulent sputum (King et al. 2006a). In addition to chronic respiratory symptoms, frequent exacerbations associated with bacterial infection and airflow obstruction result in a poor quality of life (Martinez-Garcia et al. 2005) and chronic morbidity and mortality (Keistinen et al. 1997; Chan-Yeung et al. 2008). Goals of bronchiectasis treatment are to identify underlying treatable causes of bronchiectasis and to reduce acute exacerbation and improve quality of life. To achieve these goals, multimodality treatments has been tried including antimicrobial therapy, anti-inflammatory medications, airway clearance and resectional surgery (O'Donnell 2008).
Despite its clinical importance as a chronic respiratory disease, the prevalence of bronchiectasis is not well-defined. Studies suggest that both the incidence and prevalence are declining in developed countries (O’Donnell 2008). In a recent retrospective analysis of the healthcare claims database in US, the prevalence of bronchiectasis was 4.2 per 100,000 persons in subjects aged $18-34$ years, and 271.8 per 100,000 among those aged more than 75 years (Weycker et al. 2005). Compared with the prevalence in the US, a higher prevalence was reported in New Zealand children (Edwards et al. 2003) and Alaskan native children (Singleton et al. 2000). According to the 1990 Hong Kong Government statistics, the hospital admission rate was 16.4 per 100,000 persons (Tsang and Tipoe 2004), which suggests that the prevalence in Asian countries might be higher than in other areas of the world. Comparison and estimation of the prevalence of bronchiectasis is limited, because the previous studies are heterogeneous in the definition of bronchiectasis; in addition, enrolled cases were

Received October 5, 2010; revision accepted for publication November 2, 2010. doi: 10.1620/tjem.222.237

Correspondence: Sang-Heon Kim, M.D., Ph.D., Department of Internal Medicine, Hanyang University College of Medicine, 17

Haengdang-dong, Seongdong-gu, Seoul 133-792, Korea.

e-mail: sangheonkim@hanyang.ac.kr 
restricted to patients who required healthcare utilization for their symptoms and/or acute exacerbation. Some studies used medical insurance records of reported cases (Weycker et al. 2005), while others defined bronchiectasis as involving cases referred to tertiary care centers (Singleton et al. 2000; Edwards et al. 2003). Therefore, the prevalence of bronchiectasis was likely to be underestimated. Epidemiologic studies are needed to quantitatively estimate the prevalence among general populations and describe the clinical features.

Diagnosis of bronchiectasis is confirmed by high resolution computed tomography (HRCT), which is both sensitive and specific in detecting pathologically dilated bronchi (Grenier et al. 1986). Therefore, we aimed to evaluate the prevalence of bronchiectasis based on computed tomography (CT) findings, the gold standard for the diagnosis of bronchiectasis, in adult subjects who underwent a health screening program and chest CT. In addition, we assessed demographic and clinical characteristics of bronchiectasis and identified risk factors for bronchiectasis by retrospective analysis of electronic medical records of the study subjects.

\section{Methods}

Subjects

We retrospectively analyzed the electronic medical records and the chest CT findings of the study subjects who participated in the health screening program at Hanyang University Hospital Health Promotion Center, Seoul, Korea, from January to December 2008. Adult subjects, aged 23-86, participated in the health screening program to determine their current health status and detect any illness in the early stage. Because chest CT scans were optional, the decision belonged to the subjects and was made before consulting physicians. Generally, chest CT was selected by the subjects for early detection of lung cancer, which might not be seen on simple chest radiographs. During the study period, $1,409(24.6 \%)$ of 5,727 participants in the screening program underwent chest CT scans. All subjects in the health screening program were asked to complete questionnaires regarding age, sex, smoking status, previously diagnosed diseases including asthma and pulmonary tuberculosis (TB), and current symptoms including cough, sputum, dyspnea, and hemoptysis. The questionnaires were retrieved from 95 subjects $(73.5 \%)$ with bronchiectasis and 1,118 subjects $(80.0 \%)$ without bronchiectasis. Spiro- metry, which was also optional, was selected based on the subject's decision before medical consultation. Obstructive abnormality of the spirometry was determined by the ratio of forced expiratory volume in $1 \mathrm{~s}\left(\mathrm{FEV}_{1}\right)$ over forced vital capacity $(\mathrm{FVC})$. This study was approved by the Institutional Review Board.

\section{Diagnostic criteria of bronchiectasis}

The diagnosis of bronchiectasis was made if there was abnormal bronchial dilatation in any area of both lungs on chest CT, regardless of respiratory symptoms. Abnormal bronchial dilatation was defined as the internal diameter of the bronchus being greater than the diameter of its adjacent pulmonary artery and failure of the bronchi to taper (McGuinness and Naidich 2002). We assessed the locations of bronchiectasis in both lungs and involved lobes.

\section{Statistical analysis}

Group characteristics were compared using the chi-square test for categorical variables and the Student's $t$-test for continuous variables. Risk factors for the development of bronchiectasis were determined by multivariate logistic regression analysis adjusted for age, gender, smoking status, diagnosed asthma, and history of pulmonary TB. These variables were selected a priori. Statistical analyses were performed using SPSS (version 17.0; SPSS, Chicago, IL, USA), and $p$-values $<0.05$ were considered statistically significant.

\section{Prevalence of bronchiectasis}

Of 1,409 studied subjects (aged 23-86) who were screened for respiratory diseases using chest CT for 12 months, $129(9.1 \%)$ were identified as having bronchiectasis. The prevalence of bronchiectasis was higher in females than in males $(11.5 \%$ vs. $7.9 \%, p=0.022$, a univariate analysis, Table 1). In addition, the prevalence tended to increase with age (Fig. 1). In subjects aged $\geq 70$ years, the prevalence $(20.4 \%)$ was higher than in the other age groups. Smoking status of subjects with bronchiectasis was not different from those without bronchiectasis. The proportion of subjects with a previous history of pulmonary TB was significantly higher in patients with bronchiectasis than in those without bronchiectasis $(15.8 \%$ vs. $3.4 \%, p<0.001$, a univariate analysis). However, there was no significant difference in proportions of physician-diagnosed asthma

Table 1. Characteristics of bronchiectasis.

\begin{tabular}{lccc}
\hline \multicolumn{1}{c}{ Characteristics } & $\begin{array}{c}\text { Bronchiectasis } \\
(n=129)\end{array}$ & $\begin{array}{c}\text { Non-Bronchiectasis } \\
(n=1,280)\end{array}$ & $p$ value \\
\hline Age, years, mean \pm s.D. & $54.9 \pm 10.5$ & $50.1 \pm 10.2$ & $<0.001$ \\
Female sex, \% $(n)$ & $45.0(58)$ & $34.9(447)$ & 0.022 \\
Smoking status & & & NS \\
$\quad$ Current smoker, \% $(n)$ & $25.2(24)$ & $28.5(341)$ & \\
Former smoker, \% $(n)$ & $28.4(27)$ & $24.1(277)$ & \\
Never smoker, \% $(n)$ & $46.3(44)$ & $46.3(533)$ & NS \\
Diagnosed asthma, \% $(n)$ & $2.1(2)$ & $1.3(15)$ & $<0.001$ \\
History of pulmonary TB, \% $(n)$ & $15.8(15)$ & $3.4(39)$ & \\
\hline
\end{tabular}

NS, not significant 


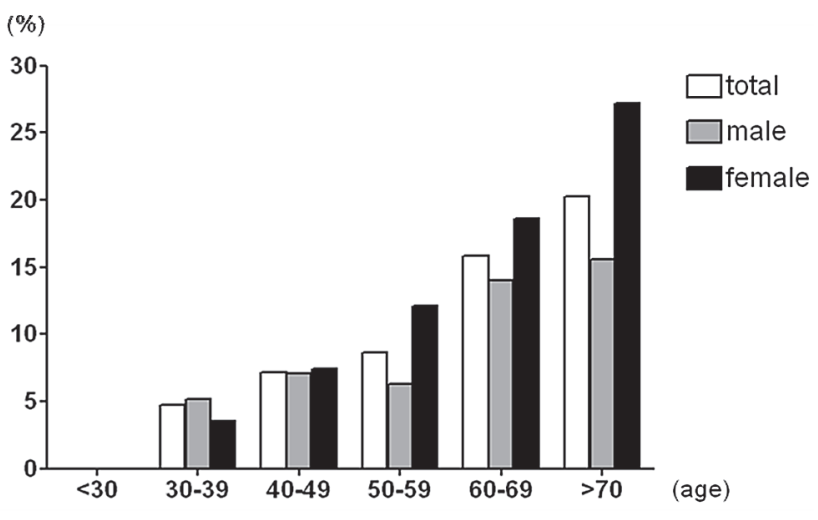

Fig. 1. Prevalence of bronchiectasis according to age groups. Empty bars indicate the prevalence in total subjects. Gray bars indicate prevalence in males, and filled bars represent prevalence in females.

Table 2. Respiratory symptoms in subjects with bronchiectasis $(n=95)$.

\begin{tabular}{lc}
\hline Respiratory symptoms & $\%(n)$ \\
\hline Cough and/or sputum & $37.9(36)$ \\
Dyspnea on exertion & $26.3(25)$ \\
Dyspnea at rest & $7.4(7)$ \\
Hemoptysis & $1.0(1)$ \\
\hline
\end{tabular}

Table 3. Spirometry in subjects with bronchiectasis $(n=$ 47).

\begin{tabular}{lc}
\hline \multicolumn{1}{c}{ Characteristics } & Mean \pm s.D. \\
\hline $\mathrm{FEV}_{1}, \mathrm{~L}$ & $2.4 \pm 0.7$ \\
$\mathrm{FEV}_{1}, \%$ predicted & $98.7 \pm 12.5$ \\
$\mathrm{FVC}, \mathrm{L}$ & $3.0 \pm 0.7$ \\
$\mathrm{FVC}, \%$ predicted & $97.5 \pm 11.5$ \\
$\mathrm{FEV}_{1} / \mathrm{FVC}, \%$ & $80.9 \pm 9.6$ \\
\hline
\end{tabular}

between the two groups.

\section{Clinical characteristics of bronchiectasis}

Next, we evaluated respiratory symptoms in the study subjects, who responded to the questionnaire $(95$ subjects with bronchiectasis and 1,151 subjects without bronchiectasis). Respiratory symptoms were present in only $53.7 \%$ of subjects with bronchiectasis, while $23.6 \%$ of subjects without bronchiectasis reported any one of the respiratory symptoms. In subjects with bronchiectasis, the most common symptoms were cough and/or sputum (37.9\%), followed by dyspnea on exertion (26.3\%), dyspnea at rest $(7.4 \%)$, and hemoptysis $(1.0 \%)$ (Table 2). Spirometry was performed in 47 (36.4\%) patients with bronchiectasis, and there was no significant airflow obstruction $\left(\mathrm{FEV}_{1} / \mathrm{FVC}\right.$ $<0.7)$ in most patients except one person. Mean values of percent predicted $\mathrm{FEV}_{1}$ and $\mathrm{FVC}$ were $98.7 \pm 12.5$ and 97.5 \pm 11.5 , respectively (Table 3 ). The mean ratio of $\mathrm{FEV}_{1} /$
Table 4. Chest CT findings in subjects with bronchiectasis $(n=129)$.

\begin{tabular}{cc}
\hline Chest CT presentation & $\%(n)$ \\
\hline Involvement of lobes & \\
Left upper lobe & $36.4(47)$ \\
Right lower lobe & $33.3(43)$ \\
Right middle lobe & $30.2(39)$ \\
Left lower lobe & $28.7(37)$ \\
Right upper lobe & $24.0(31)$ \\
Number of involved lobes & \\
1 & $62.0(80)$ \\
2 & $26.4(34)$ \\
3 & $7.0(9)$ \\
4 & $2.3(3)$ \\
5 & $1.6(2)$ \\
\hline
\end{tabular}

Table 5. Risk factors for bronchiectasis by multivariate logistic regression analysis $(n=1,246)$.

\begin{tabular}{lcc}
\hline & $p$ value & OR $(95 \%$ CI $)$ \\
\hline Age & 0.001 & $2.49(1.56-3.98)$ \\
Female sex & 0.733 & $1.12(0.59-1.92)$ \\
Smoking status & 0.917 & $1.03(0.56-1.92)$ \\
Diagnosed asthma & 0.819 & $1.21(0.25-5.92)$ \\
History of pulmonary TB & 0.001 & $4.61(2.39-8.88)$ \\
\hline
\end{tabular}

FVC was $80.9 \pm 9.6$. The most frequently involved sites of bronchiectasis were the left upper lobe (36.4\%) and right lower lobe $(33.3 \%)$ (Table 4$)$. More than $37.5 \%$ of cases showed involvement of two or more lobes.

\section{Risk factors of bronchiectasis}

In a multivariate logistic regression analysis for the development of bronchiectasis, age (OR 2.49, 95\% CI 1.56$3.98, p=0.001)$ and history of pulmonary TB (OR 4.61, $95 \%$ CI $2.39-8.88, p=0.001$ ) were found to significantly increase the risk of bronchiectasis (Table 5). Gender, smoking status, and asthma were not independently associated with the risk of bronchiectasis.

\section{Discussion}

The prevalence of bronchiectasis in general populations has not been determined, especially in non-western, underdeveloped countries. In the present retrospective study in healthy Korean adults who underwent a health screening and chest CT, we found a very high prevalence of bronchiectasis. Among those patients with bronchiectasis, a high proportion was asymptomatic. In addition, the presence of bronchiectasis was significantly associated with age and previous history of pulmonary TB. These findings suggest that the real prevalence of bronchiectasis might be much higher than the previous estimation, and that most patients remain undetected. 
The estimated prevalence of bronchiectasis in our study was markedly higher than in previous reports of adults and children from other populations. The differences might be due to dissimilarity in the study design, study populations, or definition or diagnostic criteria of bronchiectasis. Most previous studies estimated the prevalence rate of bronchiectasis from identified cases in health-care claims (Weycker et al. 2005) or medical records at tertiary care hospitals in a community (Singleton et al. 2000; Edwards et al. 2003). Although cases enrolled in these analyses were confirmed using HRCT, the gold standard for diagnosing bronchiectasis (Ooi et al. 2002), only patients with severe bronchiectasis requiring medical evaluation or health care utilization were enrolled as cases; those with asymptomatic or mild symptoms may not have been evaluated using HRCT, resulting in undetected bronchiectasis cases and underestimation of the prevalence of bronchiectasis. A growing body of evidence shows that the true prevalence of chronic respiratory diseases such as COPD, when evaluated using objective diagnostic methods, is much higher than prevalence estimated by reported or physician-diagnosed cases (Kim et al. 2005). To overcome this limitation in previous studies, and estimate the true prevalence of bronchiectasis, the same diagnostic method should be performed in all study populations. However, it is impractical to apply HRCT to a general population for assessment of the prevalence of bronchiectasis. Therefore, we chose the health screening examinees for the first study subjects in the evaluation of bronchiectasis because they underwent chest $\mathrm{CT}$ for early detection of lung diseases, such as lung cancer. The health screening examinees had some limitations as study subjects in that patients with severe respiratory diseases, including bronchiectasis, were not likely to be included in the study population; in addition, only those who could afford the expense of chest CT might be enrolled. Although they are not representative of the general population, we believe that the results obtained from evaluation of the health screening examinees may reflect the prevalence in the general population.

Although HRCT scanning is widely used for the diagnosis of bronchiectasis (Grenier et al. 1986), the subjects in this study were evaluated using conventional spiral CT because they wanted to be examined for the presence of lung cancer. Using a different CT protocol may not have detected the lesions and may have missed the cases of bronchiectasis. However, spiral CT was shown to be as effective and reliable in the diagnosis and assessment of bronchiectasis as HRCT (van der Bruggen-Bogaarts et al. 1996; Jung et al. 2000). Therefore, it is less likely that a significant number of patients with bronchiectasis went undetected or were misdiagnosed with the use of conventional spiral CT.

Demographic characteristics of the patients with bronchiectasis in our study are similar to previous findings (older age and more common in females) (Nicotra et al. 1995; Pasteur et al. 2000; Weycker et al. 2005; King et al. 2006b). One of the more interesting findings in this study was that the proportion of asymptomatic patients with bronchiectasis was very high-up to $46 \%$. The presence of asymptomatic bronchiectasis seems to have gone unnoticed in previous studies because most cases were diagnosed in those who presented for chronic respiratory symptoms (Pasteur et al. 2000; King et al. 2006b). It is not known whether these asymptomatic patients may progress to symptomatic status in the future. Studies on the clinical courses and prognosis of asymptomatic bronchiectasis should be performed. In symptomatic patients, frequencies of respiratory symptoms are comparable to previous reports (King et al. 2006a). Spirometry revealed no significant decrease in lung function in our study population. These findings differ from previous observations in symptomatic bronchiectasis, which often included mild airway obstruction (Pasteur et al. 2000; King et al. 2006a, 2006b; Martinez-Garcia et al. 2007). We speculate that the difference might be resulted from the difference in the study designs and studied populations. Because we enrolled adult subjects who underwent health screening program, it is likely that the most patients with bronchiectasis in the current study are those with mild severity or subclinical status. Thus, the notable characteristics of patients with bronchiectasis in our study can be summarized as a high proportion of asymptomatic subjects and preserved lung function. These findings suggest that true clinical features of bronchiectasis are milder than those observed in previous studies.

While idiopathic cases are most common, several risk factors and etiologies for the development of bronchiectasis were identified in previous studies on symptomatic bronchiectasis (Nicotra et al. 1995; Pasteur et al. 2000; Shoemark et al. 2007): postinfectious complications, mucociliary clearance defects associated with genetic diseases, immune deficiency, and chronic inflammatory conditions such as rheumatoid arthritis and inflammatory bowel disease. Of these, infectious diseases, including pulmonary TB, pertussis, measles, and nontuberculous Mycobacterial infection, were most frequently associated with the risk of developing bronchiectaisis. We found that pulmonary TB and age were independent risk factors for the development of bronchiectasis in the study population using a multivariate logistic regression analysis. In addition, there was no close relation between age and the previous TB infection and the proportions of subjects with the previous history of TB were similar between age groups. Although the association of TB with bronchiectasis has been known for a long time (Jones et al. 1950; Salkin 1950), the relative contribution of TB to the development of bronchiectasis varies from 1-32\%, depending on study populations (Jordan et al. 2010). Contrary to the low prevalence of TB in western countries, many Asian countries, including Korea, show a high incidence rate (Frieden et al. 2003). Although decreasing, the prevalence of TB in Korea was very high up to over 1,000 active cases of 100,000 subjects in the nationwide survey in 1995 (Hong et al. 1998). In a study on the Thai population, TB was identified as the most common 
etiology for bronchiectasis (32\% of patients) (Palwatwichai et al. 2002). The association of TB with bronchiectasis could explain the very high prevalence of bronchiectasis in our population. About fifteen percent of patients with bronchiectasis reported a previous history of pulmonary TB; among those who did not report a previous history of pulmonary $\mathrm{TB}$, we assume that a significant number may have experienced pulmonary TB with resultant bronchiectasis. While the lower lobes were the most frequently involved areas in the previous reports on symptomatic bronchiectasis (Karadag et al. 2005; King et al. 2006b), the current study revealed that both upper lobes and the right middle lobe were very commonly affected. Because upper lobe bronchiectasis is frequently related to pulmonary TB (Salkin 1950), these findings also suggest that bronchiectasis in our population is significantly associated with pulmonary TB. Due to the lack of information in our retrospective study, we could not determine the roles of other infectious diseases, immune deficiency, and other inflammatory diseases in the development of bronchiectasis. Future studies should be performed on the associations of these etiologies with asymptomatic bronchiectasis.

In conclusion, we found that the prevalence of bronchiectasis in health screening examinees was very high based on chest CT findings. Many patients were asymptomatic. Age and a previous history of TB were identified as independent risk factors for the development of bronchiectasis. These findings suggest that the real prevalence of bronchiectasis is much higher than the previous estimation. Further studies are needed to evaluate the clinical characteristics and prognosis of unnoticed asymptomatic bronchiectasis.

\section{Acknowledgments}

This study was supported by the research fund of Hanyang University (HY-2006-N).

\section{Declaration of Interest}

The authors report no conflicts of interest.

\section{References}

Barker, A.F. (2002) Bronchiectasis. N. Engl. J. Med., 346, 13831393.

Chan-Yeung, M., Lai, C.K., Chan, K.S., Cheung, A.H., Yao, T.J., Ho, A.S., Ko, F.W., Yam, L.Y., Wong, P.C., Tsang, K.W., Lam, W.K., Ho, J.C., Chu, C.M., Yu, W.C., Chan, H.S., Ip, M.S., Hui, D.S. \& Tam, C.Y. (2008) The burden of lung disease in hong kong: a report from the hong kong thoracic society. Respirology, 13 Suppl 4, S133-165.

Edwards, E.A., Asher, M.I. \& Byrnes, C.A. (2003) Paediatric bronchiectasis in the twenty-first century: experience of a tertiary children's hospital in New Zealand. J. Paediatr. Child Health, 39, 111-117.

Frieden, T.R., Sterling, T.R., Munsiff, S.S., Watt, C.J. \& Dye, C. (2003) Tuberculosis. Lancet, 362, 887-899.

Grenier, P., Maurice, F., Musset, D., Menu, Y. \& Nahum, H. (1986) Bronchiectasis: assessment by thin-section CT. Radiology, 161, 95-99.

Hong, Y.P., Kim, S.J., Lew, W.J., Lee, E.K. \& Han, Y.C. (1998) The seventh nationwide tuberculosis prevalence survey in
Korea, 1995. Int. J. Tuberc. Lung Dis., 2, 27-36.

Jones, E.M., Peck, W.M., Woodruff, C.E. \& Willis, H.S. (1950) Relationships between tuberculosis and bronchiectasis; a study of clinical and of post-mortem material. Am Rev Tuberc, 61, 387-398.

Jordan, T.S., Spencer, E.M. \& Davies, P. (2010) Tuberculosis, bronchiectasis and chronic airflow obstruction. Respirology, 15, 623-628.

Jung, K.J., Lee, K.S., Kim, S.Y., Kim, T.S., Pyeun, Y.S. \& Lee, J.Y. (2000) Low-dose, volumetric helical CT: image quality, radiation dose, and usefulness for evaluation of bronchiectasis. Invest. Radiol., 35, 557-563.

Karadag, B., Karakoc, F., Ersu, R., Kut, A., Bakac, S. \& Dagli, E. (2005) Non-cystic-fibrosis bronchiectasis in children: a persisting problem in developing countries. Respiration, $\mathbf{7 2}$, 233-238.

Keistinen, T., Saynajakangas, O., Tuuponen, T. \& Kivela, S.L. (1997) Bronchiectasis: an orphan disease with a poorly-understood prognosis. Eur. Respir. J., 10, 2784-2787.

Kim, D.S., Kim, Y.S., Jung, K.S., Chang, J.H., Lim, C.M., Lee, J.H., Uh, S.T., Shim, J.J. \& Lew, W.J. (2005) Prevalence of chronic obstructive pulmonary disease in Korea: a populationbased spirometry survey. Am. J. Respir. Crit. Care Med., 172, 842-847.

King, P., Holdsworth, S., Freezer, N. \& Holmes, P. (2006a) Bronchiectasis. Intern Med J, 36, 729-737.

King, P.T., Holdsworth, S.R., Freezer, N.J., Villanueva, E. \& Holmes, P.W. (2006b) Characterisation of the onset and presenting clinical features of adult bronchiectasis. Respir. Med., 100, 2183-2189.

Martinez-Garcia, M.A., Perpina-Tordera, M., Roman-Sanchez, P. \& Soler-Cataluna, J.J. (2005) Quality-of-life determinants in patients with clinically stable bronchiectasis. Chest, 128, 739745.

Martinez-Garcia, M.A., Soler-Cataluna, J.J., Perpina-Tordera, M., Roman-Sanchez, P. \& Soriano, J. (2007) Factors associated with lung function decline in adult patients with stable noncystic fibrosis bronchiectasis. Chest, 132, 1565-1572.

McGuinness, G. \& Naidich, D.P. (2002) CT of airways disease and bronchiectasis. Radiol. Clin. North Am., 40, 1-19.

Nicotra, M.B., Rivera, M., Dale, A.M., Shepherd, R. \& Carter, R. (1995) Clinical, pathophysiologic, and microbiologic characterization of bronchiectasis in an aging cohort. Chest, 108, 955-961.

O'Donnell, A.E. (2008) Bronchiectasis. Chest, 134, 815-823.

Ooi, G.C., Khong, P.L., Chan-Yeung, M., Ho, J.C., Chan, P.K., Lee, J.C., Lam, W.K. \& Tsang, K.W. (2002) High-resolution CT quantification of bronchiectasis: clinical and functional correlation. Radiology, 225, 663-672.

Palwatwichai, A., Chaoprasong, C., Vattanathum, A., Wongsa, A. \& Jatakanon, A. (2002) Clinical, laboratory findings and microbiologic characterization of bronchiectasis in thai patients. Respirology, 7, 63-66.

Pasteur, M.C., Helliwell, S.M., Houghton, S.J., Webb, S.C., Foweraker, J.E., Coulden, R.A., Flower, C.D., Bilton, D. \& Keogan, M.T. (2000) An investigation into causative factors in patients with bronchiectasis. Am. J. Respir. Crit. Care Med., 162, 1277-1284.

Salkin, D. (1950) Tuberculosis as a cause of upper lobe bronchiectasis. Calif. Med., 73, 577-580

Shoemark, A., Ozerovitch, L. \& Wilson, R. (2007) Aetiology in adult patients with bronchiectasis. Respir. Med., 101, 11631170.

Singleton, R., Morris, A., Redding, G., Poll, J., Holck, P., Martinez, P., Kruse, D., Bulkow, L.R., Petersen, K.M. \& Lewis, C. (2000) Bronchiectasis in Alaska native children: causes and clinical courses. Pediatr. Pulmonol., 29, 182-187.

Tsang, K.W. \& Tipoe, G.L. (2004) Bronchiectasis: not an orphan disease in the east. Int. J. Tuberc. Lung Dis., 8, 691-702. 
van der Bruggen-Bogaarts, B.A., van der Bruggen, H.M., van Waes, P.F. \& Lammers, J.W. (1996) Assessment of bronchiectasis: comparison of HRCT and spiral volumetric CT. J. Comput. Assist. Tomogr., 20, 15-19.
Weycker, D., Edelsberg, J., Oster, G. \& Tino, G. (2005) Prevalence and economic burden of bronchiectasis. Clin. Pulm. Med., 12, 205-209. 